\title{
Improving the Yule-Nielsen modified spectral Neugebauer model by dot surface coverages depending on the ink superposition conditions
}

\author{
Roger David Hersch, Frédérique Crété \\ Ecole Polytechnique Fédérale de Lausanne (EPFL), Switzerland
}

\begin{abstract}
Dot gain is different when dots are printed alone, printed in superposition with one ink or printed in superposition with two inks. In addition, the dot gain may also differ depending on which solid ink the considered halftone layer is superposed. In a previous research project, we developed a model for computing the effective surface coverage of a dot according to its superposition conditions. In the present contribution, we improve the Yule-Nielsen modified Neugebauer model by integrating into it our effective dot surface coverage computation model. Calibration of the reproduction curves mapping nominal to effective surface coverages in every superposition condition is carried out by fitting effective dot surfaces which minimize the sum of square differences between the measured reflection density spectra and reflection density spectra predicted according to the Yule-Nielsen modified Neugebauer model. In order to predict the reflection spectrum of a patch, its known nominal surface coverage values are converted into effective coverage values by weighting the contributions from different reproduction curves according to the weights of the contributing superposition conditions. We analyze the colorimetric prediction improvement brought by our extended dot surface coverage model for clustered-dot offset prints, thermal transfer prints and ink-jet prints. The color differences induced by the differences between measured reflection spectra and reflection spectra predicted according to the new dot surface estimation model are quantified on 729 different cyan, magenta, yellow patches covering the full color gamut. As a reference, these differences are also computed for the classical Yule-Nielsen modified spectral Neugebauer model incorporating a single halftone reproduction curve for each ink. Taking into account dot surface coverages according to different superposition conditions considerably improves the predictions of the Yule-Nielsen modified Neugebauer model. In the case of offset prints, the mean difference between predictions and measurements expressed in CIE-LAB CIE-94 $\Delta E_{94}$ values is reduced at 100 lpi from 1.54 to 0.90 (accuracy improvement factor: 1.7) and at 150 lpi it is reduced from 1.87 to 1.00 (accuracy improvement factor: 1.8). Similar improvements have been observed for a thermal transfer printer at $600 \mathrm{dpi}$, at lineatures of 50 and 75 lpi. In the case of an ink-jet printer at $600 \mathrm{dpi}$, the mean $\Delta E_{94}$ value is reduced at 75 lpi from 3.03 to 0.90 (accuracy improvement factor: 3.4) and at $100 \mathrm{lpi}$ from 3.08 to 0.91 (accuracy improvement factor: 3.4).
\end{abstract}

Keywords: Color printing, color halftone, spectral prediction model, Yule-Nielsen modified spectral Neugebauer model, dot gain, ink spreading, ink superposition conditions, effective surface coverages, tone reproduction curves.

\section{INTRODUCTION}

Since more than 50 years, attempts are being made to build models predicting the color of printed halftone images. To offer accurate predictions, the models need to take into account, at least to some extend, the phenomena determining the interactions of inks and paper and of light and halftone prints.

Many different phenomena influence the reflection spectrum of a color halftone patch printed on a diffusely reflecting substrate (e.g. paper). These phenomena comprise the surface (Fresnel) reflection at the interface between the air and the paper, light scattering and reflection within the substrate (i.e. the paper bulk), and the internal (Fresnel) reflections at the interface between the paper and the air. The lateral scattering of light within the paper substrate and the internal reflections at the interface between the paper and the air are responsible for what is generally called the optical dot gain, known as the Yule-Nielsen effect. 
In addition, due to the printing process, the deposited ink surface coverage is generally larger than the nominal coverage, yielding a "physical" dot gain (also called "mechanical" dot gain). Effective ink surface coverages depend on the inks, on the paper, and also on the specific superpositions of an ink halftone and the solid inks.

At the present time, according to the literature [1], [2], [3], among the existing spectral reflection prediction models (see section 2), mainly the well-known Yule-Nielsen modified spectral Neugebauer model [4], [5] seems to be usable in practice. Most other spectral prediction models (see section 2) allow to explore various effects, but are either too complex or not accurate and comprehensive enough to be usable in practice.

In the present contribution, we improve the Yule-Nielsen modified Neugebauer model by applying the effective dot surface coverage computation model that we developed within the framework of a new spectral prediction model for printing color images with metallic inks [8], [9]. Effective dot surfaces are fitted separately for every superposition condition by minimizing the sum of square differences between measured reflection density spectra and reflection density spectra predicted according to the Yule-Nielsen modified Neugebauer model. This yields, for each superposition condition, a reproduction curve mapping nominal to effective surface coverages. At prediction time, nominal coverages values are converted into effective coverage values by weighting the contributions from different reproduction curves according to the weights of the contributing superposition conditions.

The $n$-value of the Yule-Nielsen modified Neugebauer model for a given printer and screen element frequency is obtained by computing for all or for a subset of the considered color samples the mean CIELAB $\Delta E_{94}$ color difference [10] between predicted and measured reflection spectra. This mean color difference represents a measure of quality for the predictions obtained with a given $n$-value. By iterating across possible $n$-values, one selects the $n$-value yiedling the lowest minimal color difference between predicted and measured reflection spectra.

The benefit of the new dot coverage model is verified by comparing measured color halftone patch spectra and predicted reflection spectra, for 729 patches, produced by generating all combinations of inks at nominal coverages $0 \%, 13 \%, 25 \%, 38 \%, 50 \%, 63 \%, 75 \%, 88 \%$ and $100 \%$. We quantify the visual quality of color halftone patch predictions by converting measured and predicted spectra first to CIE-XYZ and then to CIE-LAB [11, pp. 8-12]. The distance as defined by the CIE-LAB (1976) $\Delta E_{a b}$ or respectively by the CIE-94 $\Delta E_{94}$ color difference formula gives a measure of the visually perceived distance between measured and predicted spectra.

The measurements are carried out with a Gretag Eye-One photospectrometer having a $45^{\circ} / 0^{\circ}$ geometry, i.e. with a D65 light source illuminating the printed sample at an angle of $45^{\circ}$ and a sensor capturing the reflected spectrum at $0^{\circ}$ (normal to the printed sample).

\section{THE YULE-NIELSEN MODIFIED SPECTRAL NEUGEBAUER MODEL AND RELATED APPROACHES}

In early prediction models of color halftone prints, the term "dot gain" encompasses both the physical dot gain (the enlargement of the printed dot) and the optical dot gain due to the lateral propagation of light. Lateral propagation of light occurs due to scattering within the paper bulk and to internal reflections at the print-air interface. The Neugebauer model [12] predicts the CIE-XYZ tri-stimulus values of a color halftone patch as the sum of the tri-stimulus values of their individual colorants weighted by their fractional area coverages $a_{i}$. By considering instead of the tri-stimulus values of colorants their respective reflection spectra $R_{i}$, one obtains the spectral Neugebauer equations. They predict the reflection spectrum of a printed color halftone patch as a function of the reflection spectra of its individual colorants

$$
R(\lambda)=\sum_{i} a_{i} \cdot R_{i}(\lambda)
$$


In the case of independently printed cyan, magenta and yellow inks of respective coverages $c, m, y$, the fractional area coverages of the individual colorants are closely approximated by the Demichel equations (2) which give the respective surface coverages of the colorants as a function of the surface coverages of the individual inks [13]. The respective fractional areas of the colorants white, cyan, magenta, yellow, red (superposition of magenta and yellow), green (superposition of yellow and cyan), blue (superposition of magenta and cyan) and black (superposition of cyan, magenta and yellow) are respectively

white:

$$
\begin{array}{ll}
\text { white: } & a_{w}=(1-c) \cdot(1-m) \cdot(1-y) \\
\text { cyan: } & a_{c}=c \cdot(1-m) \cdot(1-y) \\
\text { magenta: } & a_{m}=(1-c) \cdot m \cdot(1-y) \\
\text { yellow: } & a_{y}=(1-c) \cdot(1-m) \cdot y \\
\text { red: } & a_{r}=(1-c) \cdot m \cdot y \\
\text { green: } & a_{g}=c \cdot(1-m) \cdot y \\
\text { blue: } & a_{b}=c \cdot m \cdot(1-y)
\end{array}
$$

cyan:

magenta:$$
\text { green: } \quad a_{g}=c \cdot(1-m) \cdot y
$$$$
\text { black: } \quad a_{k}=c \cdot m \cdot y
$$

The Neugebauer model is a generalization of the Murray-Davis model [14] whose colorants are formed by only one ink and the paper white.

Since the Neugebauer model neither takes explicitly into account the lateral propagation of light within the paper bulk nor the internal reflections (Fresnel reflections) at the paper - air interface, its predictions are not accurate [15]. Yule and Nielsen [4] modeled the non-linear relationship between the reflection spectra of paper and solid ink and the reflection spectra of single ink halftones by a power function, whose exponent $n$ is fitted according to a limited set of measured halftone patch reflection spectra. Yule proposed [6], [7] and Viggiano [5] applied the Yule-Nielsen relationship to the spectral Neugebauer equations, yielding the Yule-Nielsen modified Spectral Neugebauer model (YNSN):

$$
R(\lambda)=\left(\sum_{i} a_{i} \cdot R_{i}(\lambda)^{\frac{1}{n}}\right)^{n}
$$

This YNSN model has been used by many researchers for the characterization of printing systems [5], [2], [15], [16], [17], [18] [19]. This model seems also to play a significant role for building color management systems.

Ruckdeschel and Hauser [20] analyzed the Yule-Nielson model by modeling the lateral propagation of light within the paper bulk by a point spread function $H(x, y)$. They proposed the following expression for the reflection spectrum $R$ at position $(x, y)$

$$
R(x, y)=T(x, y) \cdot R_{p} \cdot \int_{-\infty}^{\infty} \int_{-\infty}^{\infty} T\left(x^{\prime}, y^{\prime}\right) H\left(x-x^{\prime}, y-y^{\prime}\right) d x^{\prime} d y^{\prime}
$$

Light enters at all positions $\left(x^{\prime}, y^{\prime}\right)$ located in the neighbourhood of $(x, y)$, is attenuated by the ink transmittance $T\left(x^{\prime}, y^{\prime}\right)$, enters the paper bulk, propagates laterally with a fraction $H\left(x-x^{\prime}, y-y^{\prime}\right)$ reaching position $(x, y)$, is attenuated according to the paper reflectance $R_{p}$, exits the paper bulk, is attenuated by the ink layer transmittance $T(x, y)$ and emerges at position $(x, y)$. The integral simply sums up the contributions of all light components incident at positions $\left(x^{\prime}, y^{\prime}\right)$, attenuated by $H\left(x-x^{\prime}, y-y^{\prime}\right)$, which exit at position $(x, y)$.

Much research was carried out in building models by assuming various mathematical formulations of the point spread function $H(x, y)$. Both Gaussian line spread functions [20],[21] and exponential point spread functions were proposed [22], [23], [24]. Since the point spread function can also be viewed as a probability density, probability models were proposed to describe the lateral scattering of light within the paper bulk [25], [26], [27], [28]. 
In their original paper, Yule and Nielsen [4] have shown that when scattering is inexistant, e.g. when the light scattering distance is much smaller than the screen element period, the $n$-factor is $n=1$. In the case of full scattering, i.e. when the light scattering distance is significantly larger than the screen element period, we have $n=2$. However, as underlined by Yule and Nielsen [4] and later by Rogers [29], larger $n$ factors may occur, since, due to the Fresnel reflection at the interface between the print and the air, a large part of the light emerging from the paper bulk and reaching the print-air interface is reflected back into the paper bulk [30]. This yields multiple internal reflections, which, for solid ink prints, are modeled by the Saunderson correction [31], and for halftone prints by the original Clapper-Yule model [32] or its by extensions [29], [33], [9]. Recent work has shown that in the case of ink-jet printers, $n$-factors as large as $n=10$ are reached [19], [3: private communication], probably due to the non-uniform thickness of the printed dots.

\section{PHYSICAL DOT COVERAGES ACCOUNTING FOR INK SPREADING}

Ink spreading is present when an ink halftone is printed in superposition with another solid ink or when an ink halftone is printed in superposition with two or more solid inks. In a similar manner as the physical dot gain of a single ink halftone patch printed on paper, ink spreading tends to enlarge the effective surface of a printed dot and tends to lower the resulting reflection spectrum, i.e. it yields slightly darker colors. However, effective surface coverages due to ink spreading need not be larger than the corresponding effective surface coverages of the same halftone patch printed alone on paper. Figure 1 shows examples of dot gain, defined as the effective surface coverage minus the nominal surface coverage, for an inked halftone printed alone on paper and printed in superposition with the other solid inks, in the case of clustered-dot yellow offset prints at 150 lpi (Fig. 1a) and of clustered-dot magenta prints on a thermal transfer printer at 75 lpi (Fig. 1b).

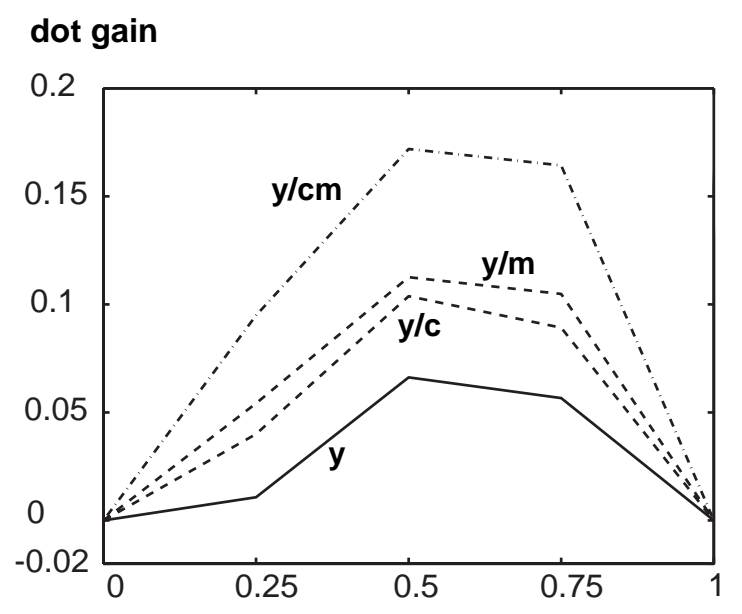

a) Clustered dot yellow halftone, offset at 150 lpi

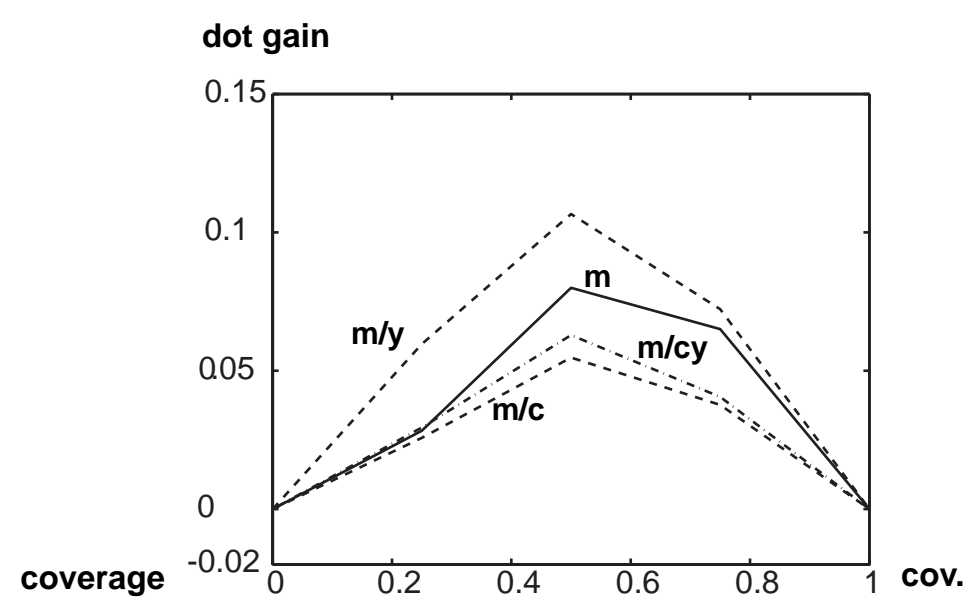

b) Clustered dot magenta halftone, thermal transfer at 75 lpi

Fig. 1 Dot gain as a function of nominal surface coverage, for ink halftones printed alone (solid line) and in superposition with the other solid inks (dotted lines), in the case (a) of an offset print and (b) of a thermal transfer print.

In the past, attempts were made to model effective surface coverages when printing overlapped ink halftones. Iino \& Berns introduced an empirical dot-gain scaling factor when an ink halftone is printed in superposition with another ink [17]. They assume that for superpositions of more than two inks, dot-gain scaling factors are multiplicative. More recently, an ink spreading model was developed which relies on the geometry of configurations of hexagonal dot overlaps [34]. These ink spreading models do not seem general enough to be applicable to a large variety of printing technologies.

The ink spreading models presented here in connection with the Yule-Nielsen modified Neugebauer model have been progressively developed by the authors, in the context of printing color images with metallic inks [8] and 
in connection with a new spectral prediction model [9] which represents an enhancement of the classical Clapper-Yule model [32]. In section 4, we present our ink spreading model where ink spreading is present only when halftones are printed on top of one or two solid inks. In section 5, we present our ink spreading model extended to halftones printed on top or below solid inks, i.e. in all superposition conditions. These effective dot surface computation models may be easily extended to 4 or more inks.

\section{INK SPREADING MODEL FOR HALFTONES PRINTED ON TOP OF OTHER INKS}

We present a first model for computing effective surface coverages (physical coverages) which accounts for ink spreading. This first model assumes that when printing two successive ink layers one on top of another, ink spreading occurs only on the top layer. The top layer does not influence the effective surface coverage of the ink layer beneath it.

Let us consider a printing process where the inks are printed by first depositing cyan, then magenta and then yellow. We therefore fit the respective ink spreaded coverages of magenta over solid (i.e. 100\%) cyan $f_{c m}(m)$, yellow over solid cyan $f_{c y}(y)$, yellow over solid magenta $f_{m y}(y)$ and yellow over solid cyan and magenta $f_{c m y}(y)$ by minimizing the square differences between measured reflection density spectra and reflection density spectra predicted according to equation (3), for a number of nominal surface coverages, e.g. $25 \%, 50 \%$, and $75 \%$. We also fit the effective coverages of each single ink halftone, respectively $f_{c}(c), f_{m}(m), f_{y}(y)$, printed alone on paper at the selected nominal coverages. The tone reproduction curves mapping nominal to effective surface coverages in the considered superposition conditions are obtained by linear interpolation between points formed by pairs of nominal coverage values and fitted effective coverage values.

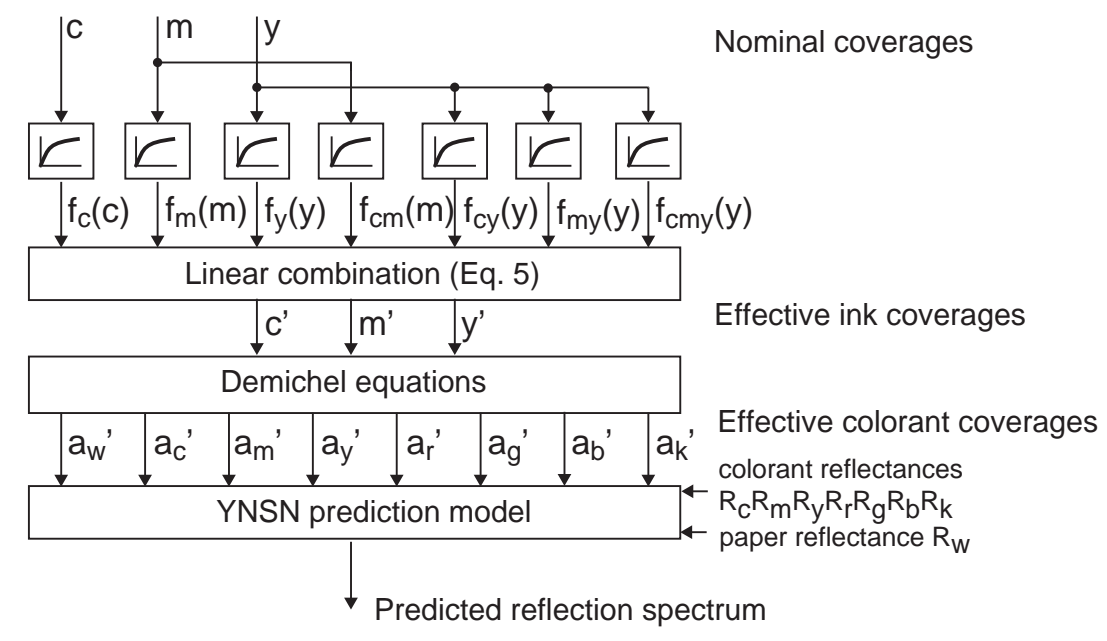

Fig. 2 YNSN spectral prediction model comprising ink spreading when an ink is printed on top of another ink

In front of our new spectral prediction model, we introduce a stage taking into account effective surface coverages induced by standard dot gain and dot gain due to ink spreading (Fig. 2). An input color with nominal coverages cmy is converted to an intermediate color with effective coverages c' $m$ ' $y$ ' obtained as a weighted sum of the effective surface coverages specific to the different superposition conditions (halftone printed alone, printed on top of a second ink and printed on top of a third ink).

The weighting coefficients are given by the respective effective colorant coverages.

$$
c^{\prime}=f_{c}(c)
$$




$$
\begin{aligned}
m^{\prime} & =f_{m}(m)\left(1-c^{\prime}\right)+f_{c m}(m) c^{\prime} \quad ; \text { magenta on top of paper and magenta on top of solid cyan (blue), } 2^{\text {nd }} \text { ink layer } \\
y^{\prime} & =f_{y}(y)\left(1-c^{\prime}\right)\left(1-m^{\prime}\right) \\
& +f_{m y}(y)\left(1-c^{\prime}\right) m^{\prime}+f_{c y}(y) c^{\prime}\left(1-m^{\prime}\right)+f_{c m y}(y) c^{\prime} m^{\prime} \\
& ; \text { yellow on top of paper, }\left(3^{\text {rd }} \text { ink layer }\right) \\
& \text { (green) and of of solid cyan \& magenta (black) }
\end{aligned}
$$

The performance of this simple ink spreading model is illustrated by the prediction accuracies in the second row ("single ink dot gain and ink spreading according to the print order") of the tables shown in the Appendix, with the corresponding optimal $n$-value of the YNSN model. The increase in prediction accuracy, compared with standard single ink dot gain optimization (i.e. one reproduction curve per ink halftone) is important. For offset prints, the prediction accuracy defined as the reciprocal of the $\Delta E_{94}$ prediction error is improved by a factor of 1.4 to 1.9 .

\section{INK SPREADING MODEL FOR ALL INK LAYER SUPERPOSITION CONDITIONS}

A more advanced model for computing the effective surface coverages in layer superpositions relies on the assumption that when a halftone layer is printed either beneath or on top of a solid layer, its effective surface coverage is modified.

We separately model as functions of nominal coverages (a) the surface coverages of single ink halftones, (b) the surface coverages of single ink halftones superposed with one solid ink and (c) the surface coverages of single ink halftones superposed with two solid inks. In order to obtain the effective coverages $\left(c^{\prime}, m^{\prime}, y^{\prime}\right)$ of the inks of a color halftone patch as a function of the nominal coverages $(c, m, y)$, we appropriately weight the contributions of the different surface coverage functions.

During calibration of the model, the functions describing effective surface coverages of single ink halftones printed in superposition with paper white, one solid ink or two solid inks are obtained by fitting effective surface coverages (e.g. at $25 \%, 50 \%$ and $75 \%$ nominal coverages) of an ink using the spectral prediction model given by equation (6). This allows to associate effective (fitted) surface coverages to the nominal surface coverages, for a limited set of halftone patches of each ink, in each ink superposition condition. By linear interpolation between the so obtained effective coverages, we obtain the functions (tone reproduction curves) mapping nominal to effective surface coverages of each ink for each ink superposition condition.

Let us consider 3 inks $i_{1}, i_{2}$ and $i_{3}$ with nominal coverages $c_{1}, c_{2}$ and $c_{3}$. The classical tone reproduction functions mapping nominal coverages to effective coverages for single ink halftones are $f_{1}\left(c_{1}\right), f_{2}\left(c_{2}\right)$ and $f_{3}\left(c_{3}\right)$. The "ink spreading" functions mapping nominal coverages of an ink to effective coverages of that ink, for single ink halftones superposed with a second solid ink and for single ink halftones superposed with two solid inks are:

- for ink $i_{1}$ of coverage $c_{1}$ superposed with solid ink $i_{2}: f_{21}\left(c_{1}\right)$,

- for ink $i_{1}$ of coverage $c_{1}$ superposed with solid ink $i_{3}: f_{31}\left(c_{1}\right)$,

- for ink $i_{2}$ of coverage $c_{2}$ superposed with solid ink $i_{1}: f_{12}\left(c_{2}\right)$,

- for ink $i_{2}$ of coverage $c_{2}$ superposed with solid ink $i_{3}: f_{32}\left(\mathrm{c}_{2}\right)$,

- for ink $i_{3}$ of coverage $c_{3}$ superposed with solid ink $i_{1}: f_{13}\left(\mathrm{c}_{3}\right)$,

- for ink $i_{3}$ of coverage $c_{3}$ superposed with solid ink $i_{2}: f_{23}\left(c_{3}\right)$,

- for ink $i_{1}$ of coverage $c_{1}$ superposed with solid inks $i_{2}$ and $i_{3}: f_{231}\left(c_{1}\right)$,

- for ink $i_{2}$ of coverage $c_{2}$ superposed with solid inks $i_{1}$ and $i_{3}: f_{132}\left(c_{2}\right)$,

- for ink $i_{3}$ of coverage $c_{3}$ superposed with solid inks $i_{1}$ and $i_{2}: f_{123}\left(c_{3}\right)$.

In the case of three inks, these 12 functions may for example be obtained by fitting 36 patches, i.e. 3 patches $(25 \%, 50 \%$ and $75 \%$ nominal coverages) per function. 
Figures $1 \mathrm{a}$ and $1 \mathrm{~b}$ give examples of dot gains obtained by fitting effective surface coverages according to the Yule-Nielsen model, for wedges printed alone, for wedges printed in superposition with one solid ink and for wedges printed in superposition with two solid inks. The effective surface coverages, and therefore the dot gains, depend if a halftone wedge is printed alone, in superposition with one ink, or in superposition with two inks. In Fig. 1a, for offset prints at 150 lpi, yellow halftones alone (y) have a smaller dot gain than yellow halftones printed in superposition with solid magenta $(\mathrm{y} / \mathrm{m})$ or solid cyan $(\mathrm{y} / \mathrm{c})$. Yellow halftones printed in superposition of solid cyan and magenta $(\mathrm{y} / \mathrm{cm})$ have the largest dot gain. However, as is shown in Fig. $1 \mathrm{~b}$ for thermal transfer prints at $75 \mathrm{lpi}$, ink spreading does not necessarily induce a larger dot gain when halftones are printed in superposition with one or two inks. In that example, magenta halftones printed in superposition with the two solid inks cyan and yellow $(\mathrm{m} / \mathrm{cy})$ have a smaller dot gain than magenta halftones printed alone.

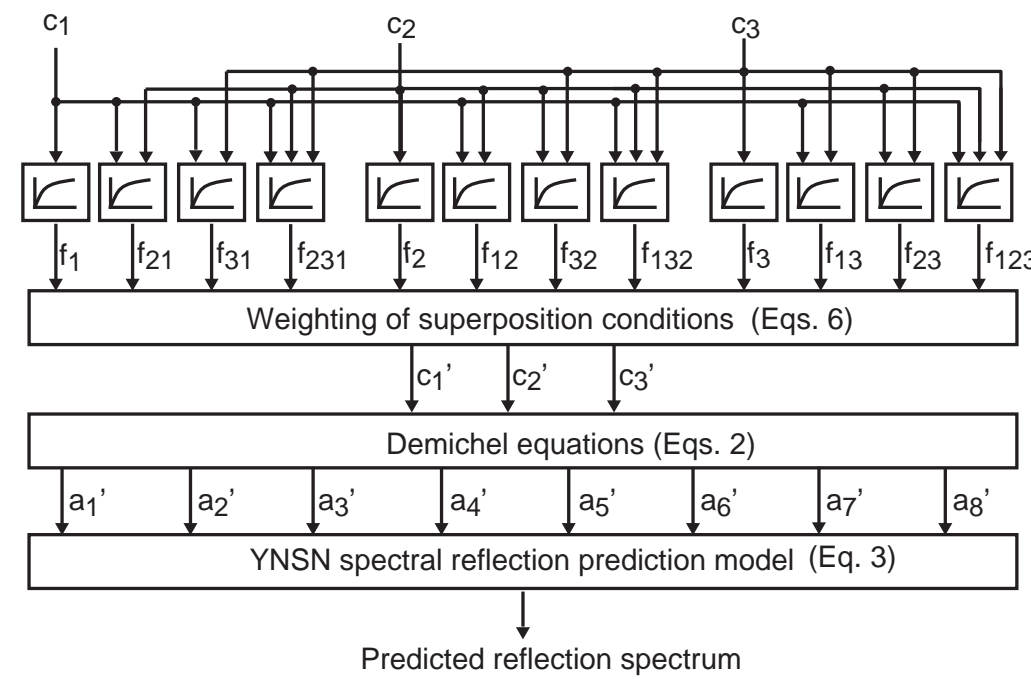

Nominal ink coverages

Tone reproduction curves

in all superposition conditions

Effective ink coverages

Effective colorant

coverages

Fig. 3 Spectral prediction model with dot gain and ink spreading in all superposition conditions

By linearly interpolating between the fitted surface coverage values, we obtain the functions (tone reproduction curves) mapping nominal to effective surface coverages for ink halftones printed in different superposition conditions.

In order to obtain the effective surface coverages $c_{1}{ }^{\prime}, c_{2}$ ' and $c_{3}$ ' of a color halftone patch, it is necessary, for each ink $i_{k}$, to weight the contributions of the corresponding mapping functions $f_{k}, f_{l k}, f_{m k}$, and $f_{l m k}(k \neq l \neq m)$. The weighting functions depend on the effective coverages of the considered ink alone, of the considered ink in superposition with a second ink and of the considered ink in superposition with the two other inks. For the considered system of 3 inks $i_{1}, i_{2}$ and $i_{3}$ with nominal coverages $c_{1}, c_{2}$ and $c_{3}$ and effective coverages $c_{1}{ }^{\prime}, c_{2}$ ' and $c_{3}{ }^{\prime}$, assuming that inks are printed independently of each other, by computing the relative weight, i.e. the relative surface of each superposition condition, we obtain the system of equations (6). In analogy with Demichel's equations (2), the proportion (relative effective surface) of a halftone patch printed with ink $i_{1}$ of coverage $c_{1}$ on paper white is $\left(1-c_{2}\right)\left(1-c_{3}^{\prime}\right)$. The proportion of the same patch printed on top of solid ink $i_{2}$ is $c_{2}$ ' $\left(1-c_{3}{ }^{\prime}\right)$, the proportion of the same patch printed on top of solid ink $i_{3}$ is $\left(1-c_{2}{ }^{\prime}\right) c_{3}$ ' and the proportion of the same patch printed on top of solid inks $i_{2}$ and $i_{3}$ is $c_{2}{ }^{\prime} c_{3}$ '. We obtain the system of equations:

$$
\begin{aligned}
& c_{1}^{\prime}=f_{1}\left(c_{1}\right)\left(1-c_{2}^{\prime}\right)\left(1-c_{3}^{\prime}\right)+f_{21}\left(c_{1}\right) c_{2}^{\prime}\left(1-c_{3}^{\prime}\right)+f_{31}\left(c_{1}\right)\left(1-c_{2}\right) c_{3}{ }^{\prime}+f_{231}\left(c_{1}\right) c_{2}^{\prime} c_{3}^{\prime} \\
& c_{2}^{\prime}=f_{2}\left(c_{2}\right)\left(1-c_{1}{ }^{\prime}\right)\left(1-c_{3}{ }^{\prime}\right)+f_{12}\left(c_{2}\right) c_{1}^{\prime}\left(1-c_{3}^{\prime}\right)+f_{32}\left(c_{2}\right)\left(1-c_{1}{ }^{\prime}\right) c_{3}{ }^{\prime}+f_{132}\left(c_{2}\right) c_{1}{ }^{\prime} c_{3}^{\prime} \\
& c_{3}^{\prime}=f_{3}\left(c_{3}\right)\left(1-c_{1}^{\prime}\right)\left(1-c_{2}^{\prime}\right)+f_{13}\left(c_{3}\right) c_{1}^{\prime}\left(1-c_{2}^{\prime}\right)+f_{23}\left(c_{3}\right)\left(1-c_{1}^{\prime}\right) c_{2}^{\prime}+f_{123}\left(c_{3}\right) c_{1}^{\prime} c_{2}^{\prime}
\end{aligned}
$$


This system of equations can be solved iteratively: one starts by setting initial values of $c_{1}$ ', $c_{2}$ ' and $c_{3}$ ' equal to the respective nominal coverages $c_{1}, c_{2}$ and $c_{3}$. After one iteration, one obtains new values for $c_{1}{ }^{\prime}, c_{2}$ ' and $c_{3}$ '. These new values are used for the next iteration. After a few iterations, typically 4 to 5 iterations, the system stabilizes and the obtained coverages $c_{1}{ }^{\prime}, c_{2}$ ' and $c_{3}$ ' are the effective coverages. The system of equations (6) allows us therefore to compute combined effective ink surface coverages (physical dot sizes) resulting from the combination of elementary ink surface coverages present in different superposition conditions. The effective colorant coverages $a_{1}{ }^{\prime}, a_{2}{ }^{\prime}, \ldots a_{8}$ ' are obtained from the effective coverages $c_{1}{ }^{\prime}, c_{2}$ ' and $c_{3}$ ' of the inks according to the Demichel equations (Eq. 2). The complete model comprising classical dot gain and ink spreading in all superposition conditions is illustrated in Fig. 3.

By taking into account the effective ink coverages (physical dot coverages) in all superposition conditions, we obtain important improvements in prediction accuracy. This is especially the case for ink-jet clustered-dot prints where predictions in respect to single ink dot gain optimization (one reproduction curve per ink halftone) are improved by a factor of 3 (see Appendix, Table 3).

\section{6. $N$-VALUE: IMPACT ON PREDICTION AND OPTIMIZATION}

The $n$-value of the Yule-Nielsen modified spectral Neugebauer model characterizes the ratio between lateral propagation of light within the paper bulk and halftone screen frequency, the multiple internal reflections between paper bulk and print-air interface as well as possible non-uniformities in the dot thickness profiles. In order to better understand the signification of the $n$-value, let us plot the mean prediction error expressed in CIELAB $\Delta E_{94}$ values as a function of increasing $n$-values, for the considered printing technologies (offset, thermal transfer and ink-jet).

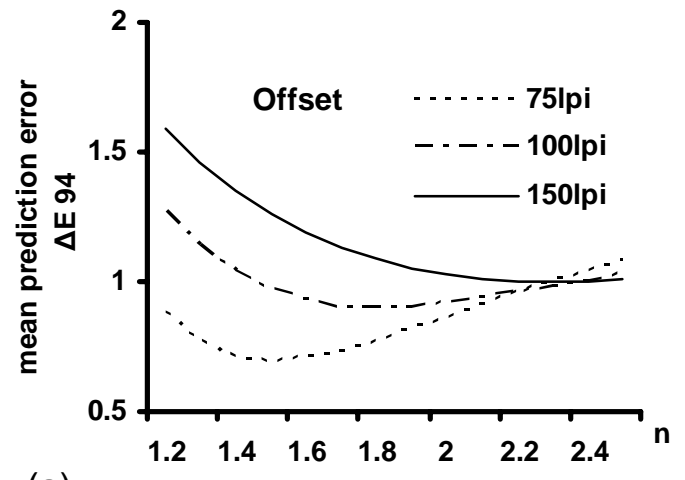

(a)

(c)

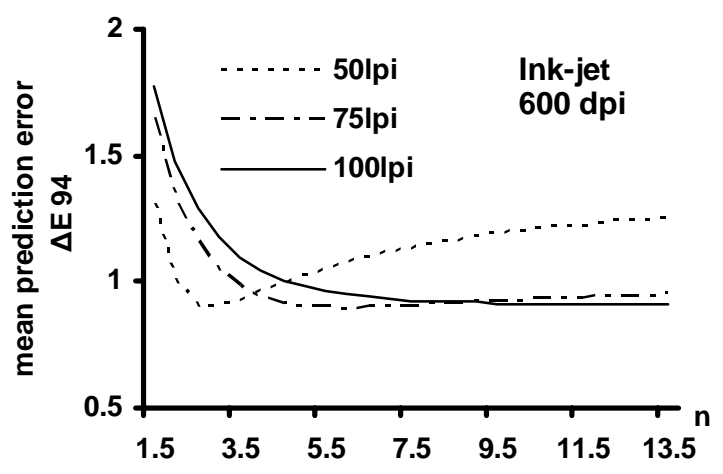

Fig. 4 Relationship between $\boldsymbol{n}$-value and mean prediction error over 729 print samples for (a) offset prints on matte coated paper at 75, 100 and $150 \mathrm{lpi}$, (b) thermal transfer prints on calendered paper at 50, 75 and 100 lpi and (c) ink-jet prints on coated paper at 50, 75 and 100 lpi 
Figs. $4 \mathrm{a}, 4 \mathrm{~b}$ and $4 \mathrm{c}$ show the mean prediction error $\left(\Delta E_{94}\right)$ as a function of the $n$-value, for the YNSN model, extended with effective dot surface coverage optimization taking into account ink spreading in all superposition conditions (section 5). The mean prediction error is obtained by computing the $\Delta E_{94}$ color difference between predicted and measured spectra, for all 729 patches distributed uniformly over the full cyan, magenta and yellow color gamut. Fig. 4 clearly shows that the optimal $n$-values increase with increasing screen frequency (lpi). However, at a high screen frequency (e.g. offset at $150 \mathrm{lpi}$ ), the mean prediction error in function of $n$-value is relatively flat, meaning that a large range of $n$-values yield a similar prediction accuracy. This may be explained by the fact that to a certain extent, variations in $n$-values may be compensated by the functions mapping nominal to effective dot surface coverages in the different superposition conditions.

Thanks to Fig. 4, one can rapidly determine the optimal $n$-value for given printing conditions, i.e. printing technology, inks, paper and lineature. However, when creating the YNSN model, the optimal $n$-value needs to be determined by relying on a small subset of print samples. In order to verify that this is feasible, let us consider as learning set for optimizing the $n$-value only the set of samples needed for performing the dot surface optimization, i.e. for establishing the tone reproduction curves. Considered models of dot surface optimization are the models relying on single ink halftones printed alone on paper ( $3 \times 3=9$ samples), ink halftones printed on top of other inks $(7 \times 3=21$ samples $)$ and ink halftones printed in all superposition conditions $(3 \times 12=36$ samples $)$. As a reference, we have also plotted the prediction accuracy for the set of all test samples ( 729 samples).

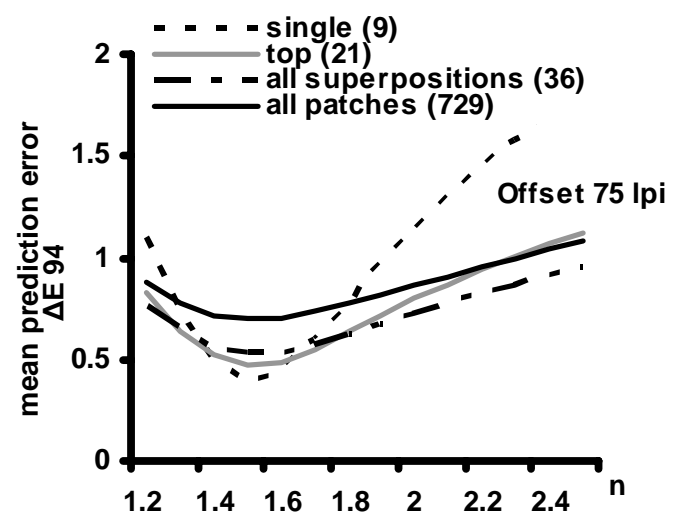

Fig. 5 Evolution of the mean prediction error in function of the $\boldsymbol{n}$-value, for learning sets comprising the samples used for establishing the functions mapping nominal to effective dot surface coverages (offset at 75lpi)

Fig. 5 shows that very similar optimal $n$-values are obtained for the different learning sets $(9$ samples, 21 samples and 36 samples) and for the set of all test samples ( 729 samples). A similar behavior has been observed for the other considered lineatures and technologies (offset, thermal transfer and ink-jet).

We can therefore conclude that for given printing conditions, a near optimal $n$-value can be determined by simply relying on the same set of measured samples as the one used for computing the functions mapping nominal to effective dot surface coverages.

\section{APPLICATION OF THE MODEL}

The proposed methods of computing effective surface coverages according to different superposition conditions were applied to offset, thermal transfer and ink-jet printing of clustered dots at various screen element frequencies, for the cyan, magenta and yellow inks. Classical clustered-dot halftoning was used, with screens mutually rotated by $30^{\circ}$. We present the prediction accuracies when printing with cyan, magenta and yellow inks on offset (Komori Lithrone 26, matte coated paper) at lineatures of 75, 100 and 150 lpi, on a thermal transfer wax printer (Alps MD-5000 at 600 dpi, calendered paper) and on an ink-jet printer (Canon BJC 8500 at 600dpi, 
coated paper) at lineatures of 50,75 and 100 lpi. The tables in the Appendix give the mean prediction errors in terms of CIELAB $1976 \Delta E_{a b}$ and CIE-94 $\Delta E_{94}$ values, the maximal prediction error and the number of patches having an error larger than $\Delta E_{a b}=4$, respectively larger than $\Delta E_{94}=3$. For fitting the effective dot surface coverages, only $25 \%, 50 \%$ and $75 \%$ nominal coverages are used, yielding in case of single ink halftones $3 \times 3=9$ patches, in case of ink spreading when printed on top of one or two inks $7 \times 3=21$ patches (Eq. 5) and in case of ink spreading for all superposition conditions $3 \times 12=36$ patches (Eq. 6). In addition, the reflectances of the paper white and of patches of all solid inks and solid ink superpositions (colorants) are measured ( 8 patches). The model is tested on 729 patches, comprising all nominal coverage combinations at $0 \%, 13 \%, 25 \%, 38 \%, 50 \%$, $63 \%, 75 \%, 88 \%$ and $100 \%$.

The prediction results clearly show that both ink spreading models improve the prediction performance. The first ink spreading model taking into account ink spreading only when one ink halftone is printed on top of a second solid ink or on top of the two other solid inks brings the most important prediction improvements for offset prints on matte coated paper. This may be explained by the fact that for offset prints on coated paper, the dot gain depends mainly if an ink halftone is printed alone or on top of other inks.

For thermal transfer and ink-jet technologies, the second ink spreading model, where all superposition cases are considered, brings the largest improvement in reflection spectra prediction accuracies. For these technologies, the dot gain depends on the specific superposition condition (e.g. the superposition of a magenta halftone and solid yellow is different from the superposition of a magenta halftone and solid cyan) rather than on the print order or the number of superposed inks.

\section{CONCLUSIONS}

Within the framework of the establishment of new spectral reflection model [9], we have developed new approaches for modeling ink spreading, a phenomenon which occurs when printing an ink halftone in superposition with one or several solid inks. In the present contribution, we extend the Yule-Nielsen modified spectral Neugebauer model [5] by incorporating a stage performing the mapping between nominal to effective dot coverages according to effective dot coverages which depend on the different ink superposition conditions.

In the first ink spreading model, we consider the ink spreading which occurs when an ink halftone is printed on top of one or two solid inks. In the second more advanced model, we generalize this concept to ink halftones printed on top or below solid inks, i.e. in superposition with solid inks. We formulate for both ink spreading models systems of equations which allow us to compute effective dot surface coverages as a weighted mean of the individual effective dot surface coverages which occur in the different superposition conditions.

For the calibration of the tone reproduction curves, i.e. the establishment of the functions mapping nominal to effective surface coverages in the different superposition conditions, effective coverage values are fitted by minimizing the sum of square differences between measured and predicted reflection density spectra. In the case of three inks (cyan, magenta and yellow), for the ink spreading model considering all superposition conditions, the calibration set comprises 44 samples. It comprises the paper white, seven solid ink samples and 36 halftone samples yielding 36 fitted (effective) surface coverages for the 12 linearly interpolating functions mapping nominal to effective surface coverages.

We have shown that the same halftone print samples used to calibrate the ink spreading model can also be used for computing for given printing conditions (print technology, lineature, inks, paper) the near optimal $n$-value of the Yule-Nielsen modified spectral Neugebauer model.

The new methods of estimating effective dot surface coverages considerably improve the predictions. Tests were carried out with 729 color patches covering the complete gamut of the output device. For offset prints at 150 lpi (Appendix, Table 1), the two ink spreading models reduce the mean prediction $\Delta E_{94}$ error between predicted and measured spectra from 1.87 to 1.00 . Therefore, they improve the prediction accuracy by a factor 
of 1.8. Errors below to $\Delta E_{94}=1$ can hardly be reduced further, since they correspond to the colorimetric variations which occur when printing identical patches at different locations of the same printed page.

For thermal transfer prints at 75 lpi (Appendix, Table 2), ink spreading in all superposition conditions reduces the mean prediction error from $\Delta E_{94}=2.00$ to $\Delta E_{94}=1.13$, i.e. the prediction accuracy is improved by a factor of 1.7 .

For ink-jet prints at 100 lpi (Appendix, Table 3), ink spreading in all superposition conditions reduces the mean prediction error from $\Delta E_{94}=3.08$ to $\Delta E_{94}=0.91$. Such a reduction in prediction error represents an improvement of prediction accuracy by a factor of 3.3 which is very significant.

For all considered technologies, we have obtained for clustered-dot halftones good to excellent reflection spectra prediction accuracies. The presented enhanced YNSN model also works for dispersed-dot and errordiffusion hafltoning techniques, but offers less prediction accuracy. Further research is needed for achieving similar prediction accuracies in respect to dispersed-dot or error-diffusion halftoning algorithms.

\section{REFERENCES}

1. D.R.Wyble, R.S. Berns, A Critical Review of Spectral Models Applied to Binary Color Printing, Journal of Color Research and Application, Vol. 25, No. 1, 4-19, (2000)

2. R. Balasubramanian, Optimization of the spectral Neugebauer model for printer characterization, Journal of Electronic Imaging, Vol. 8, No. 2, 156-166 (1999)

3. T. Ogasahara, Verification of the Predicting Model and Characteristics of Dye-Based Ink Jet Printer, Journal of Imaging Science and Technology, Vol 48, No. 2, 130-137 (2004)

4. J.A.C. Yule, W.J. Nielsen, The penetration of light into paper and its effect on halftone reproductions, Proc. TAGA, Vol. 3, 65-76 (1951),

5. J.A.S Viggiano, Modeling the Color of Multi-Colored Halftones, Proc. TAGA, 44-62 (1990)

6. J.A.C. Yule, R. Colt, Colorimetric investigations in multicolor printing, Proc. TAGA, 77-82, (1951)

7. I. Pobborovsky, M. Pearson, Computation of dot areas required to match a colorimetrically specified color using the modified Neugebauer equations, Proc. TAGA, 1972, 65-77

8. R.D. Hersch, F. Collaud, P. Emmel, Reproducing color images with embedded metallic patterns, Proc. SIGGRAPH 2003, ACM Trans. on Graphics, Vol. 22, No.3, 427-436

9. R.D. Hersch, F. Collaud, F. Crété, P. Emmel, Spectral prediction and dot surface estimation models for halftone prints, Conf. Imaging IX: Processing, Hardcopy and Applications, Proc. of SPIE-IS\&T Electronic Imaging, (Eds. R. Eschbach, G. Marcu), SPIE Vol. 5293, 356-369 (2004)

10. Commission Internationale de l'Eclairage (CIE), Industrial Color Difference Evaluation, CIE Publication No. 1161995, Central Bureau of the CIE, Vienna, 1995 (www.cie.co.at)

11. H.R. Kang, Color Technology for Electronic Imaging Devices, SPIE Optical Engineering Press (1997).

12. H.E.J. Neugebauer, Die theoretischen Grundlagen des Mehrfarbendrucks. Zeitschrift fuer wissenschaftliche Photographie, Vol. 36, 36-73, (1937), reprinted in Neugebauer Seminar on Color Reproduction, SPIE Vol-1184,194-202 (1989)

13. M.E. Demichel, 1924. Procédé, Vol. 26, 17-21.

14. A. Murray, Monochrome reproduction in photoengraving, J. Franklin Institute, Vol. 221, 721-724 (1936)

15. H.R. Kang, Applications of color mixing models to electronic printing. Journal of Electronic Imaging, Vol. 3, No. 3 , 276-287 (1994)

16. K. Iino, R.S. Berns, Building color management modules using linear optimization I. Desktop, Journal of Imaging Science and Technology, Vol. 42, No. 1, 79-94 (1998)

17. K. Iino, R.S. Berns, Building color management modules using linear optimization II. Prepress system for offset printing, Journal of Imaging Science and Technology, Vol. 42, No. 2, 99-114 (1998)

18. A. U. Agar and J. P. Allebach, "An Iterative Cellular YNSN Method for Color Printer Calibration," Proceedings of the 6th IS\&T/SID Color Imaging Conference, Scottsdale AZ, 17-20 Nov. 1998, pp. 197-200

19. S. Zuffi, R. Schettini, An innovative method for spectral-based printer characterization, Color Imaging: Device-Independent Color, Color Hardcopy, and Applications VII, Proc. of SPIE-IS\&T Electronic Imaging, Vol. 4663 (R. Esch- 
bach, G.G. Marcu eds.), 1-7 ( 2002)

20. F.R. Ruckdeschel, O.G. Hauser, Yule-Nielsen in printing: a physical analysis, Applied Optics, Vol. 17, No. 21, 33763383 (1978)

21. J.A.C. Yule, D.J. Howe, J.H. Altman, The effect of the spread function of paper on halftone reproduction, TAPPI Journal, Vol. 50, No. 7, 337-344, (1967)

22. H. Wakeshima, T. Kunishi, S. Kaneko, Light Scattering in Paper and its Effect on Halftone Reproduction, Journal of the Optical Society of America, Vol. 58, 272-273 (1968)

23. P. G. Engeldrum, B. Pridham, Application of Turbid Medium Theory to Paper Spread Function Measurements, Proc. TAGA Proc., Vol. 47, 339-352 (1995)

24. S. Gustavson, Color Gamut of Halftone Reproduction, Journal of Imaging Science and Technology, Vol. 41, No. 3, 283-290 (1997)

25. J. S. Arney, A Probability Description of the Yule-Nielsen Effect I, Journal of Imaging Science and Technology, Vol. 41, No. 6, 633-636 (1997)

26. G. Rogers, Optical Dot Gain: Lateral Scattering Probabilities, Journal of Imaging Science and Technology, Vol. 42, No. 4, 341-345, (1998)

27. L. Yang, R. Gooran, B. Kruse, Simulation of Optical Dot Gain in Multichromatic Tone Production, Journal of Imaging Science and Technology, Vol. 45, No. 2, 198-204 (2001)

28. L. Yang, R. Lenz, B. Kruse, Light scattering and ink penertration effects on tone reproduction, Journal of the Optical Society of America A, Vol. 18, No. 2, 360-366 (2001)

29. G. Rogers, A Generalized Clapper-Yule Model of Halftone Reflectance. Journal of Color Research and Application, Vol. 25, No. 6, 402-407 (2000)

30. M. Hebert, R.D. Hersch, Classical Print Reflection Models, a Radiometric Approach, Journal of Imaging Science and Technology, Vol 48, No. 4, 363-374 (2004)

31. J.L. Saunderson, Calculation of the color pigmented plastics, Journal of the Optical Society of America, Vol. 32, $727-$ 736 (1942)

32. F.R. Clapper, J.A.C Yule, The effect of multiple internal reflections on the densities of halftone prints on paper, Journal of the Optical Society of America, Vol. 43, 600-603 (1953)

33. P. Emmel, R.D. Hersch, A Unified Model for Color Prediction of Halftoned Prints, Journal of Imaging Science and Technology, Vol. 44, No. 4, 351-359 (2000)

34. P. Emmel, R.D. Hersch, Modeling ink spreading for color prediction, Journal of Imaging Science and Technology, Vol. 46, No. 3, 237-246, (2002) 


\section{APPENDIX: PREDICTION ACCURACIES}

TABLE 1. YNSN model prediction accuracy for offset cyan, magenta and yellow halftone prints

\begin{tabular}{|c|c|c|c|c|c|c|}
\hline $\begin{array}{c}\text { Offset printing (matte coated paper) } \\
729 \text { test samples }\end{array}$ & $\begin{array}{c}\operatorname{Max} \\
\Delta E_{a b}\end{array}$ & $\begin{array}{c}\text { Mean } \\
\Delta E_{a b}\end{array}$ & $\underset{\Delta E_{a b}>\mathbf{4}}{\# \text { samples }}$ & $\begin{array}{l}\operatorname{Max} \\
\Delta E_{94}\end{array}$ & $\begin{array}{l}\text { Mean } \\
\Delta E_{94}\end{array}$ & $\begin{array}{c}\text { \# samples } \\
\Delta E_{94}>\mathbf{3}\end{array}$ \\
\hline 75 lpi & \multicolumn{3}{|c|}{$\mathrm{n}=\mathbf{1 . 5}$} & \multicolumn{3}{|c|}{$\mathrm{n}=\mathbf{1 . 5}$} \\
\hline Single ink dot-gain only & 3.90 & 1.57 & 0 & 3.08 & 1.09 & 3 \\
\hline $\begin{array}{l}\text { Single ink dot gain and ink spreading } \\
\text { according to print order }\end{array}$ & 3.27 & 1.14 & 0 & 1.94 & 0.76 & 0 \\
\hline $\begin{array}{l}\text { Single ink dot gain and ink spreading for } \\
\text { all superposition conditions }\end{array}$ & 3.20 & 1.08 & 0 & 2.64 & 0.65 & 0 \\
\hline 100 lpi & \multicolumn{3}{|c|}{$n=1.8$} & \multicolumn{3}{|c|}{$n=1.8$} \\
\hline Single ink dot-gain only & 5.64 & 2.17 & 28 & 4.78 & 1.49 & 24 \\
\hline $\begin{array}{l}\text { Single ink dot gain and ink spreading } \\
\text { according to print order }\end{array}$ & 5.76 & 1.36 & 1 & 3.49 & 0.86 & 1 \\
\hline $\begin{array}{l}\text { Single ink dot gain and ink spreading for } \\
\text { all superposition conditions }\end{array}$ & 5.74 & 1.41 & 3 & 3.64 & 0.82 & 1 \\
\hline 150 lpi & \multicolumn{3}{|c|}{$\mathrm{n}=2.3$} & \multicolumn{3}{|c|}{$n=2.3$} \\
\hline Single ink dot-gain only & 7.80 & 2.79 & 121 & 5.27 & 1.80 & 60 \\
\hline $\begin{array}{l}\text { Single ink dot gain and ink spreading } \\
\text { according to print order }\end{array}$ & 4.19 & 1.51 & 4 & 2.93 & 0.94 & 0 \\
\hline $\begin{array}{l}\text { Single ink dot gain and ink spreading for } \\
\text { all superposition conditions }\end{array}$ & 4.71 & 1.50 & 14 & 2.61 & 0.86 & 4 \\
\hline
\end{tabular}

TABLE 2. Prediction accuracy for thermal transfer color halftone prints (Alps MD5000, 600 dpi)

Thermal transfer (ALPS MD-5000)

\begin{tabular}{c}
729 test samples \\
\hline $\mathbf{5 0}$ lpi \\
\hline $\begin{array}{c}\text { Single ink dot-gain only } \\
\text { Single ink dot gain and ink spreading } \\
\text { according to print order }\end{array}$
\end{tabular}

Single ink dot gain and ink spreading for \begin{tabular}{c} 
all superposition conditions \\
\hline $\mathbf{7 5}$ lpi \\
\hline $\begin{array}{c}\text { Single ink dot-gain only } \\
\text { Single ink dot gain and ink spreading } \\
\text { according to print order }\end{array}$
\end{tabular}

Single ink dot gain and ink spreading for \begin{tabular}{c} 
all superposition conditions \\
\hline $\mathbf{1 0 0}$ lpi \\
\hline Single ink dot-gain only \\
Single ink dot gain and ink spreading \\
according to print order
\end{tabular}

Single ink dot gain and ink spreading for all superposition conditions

\begin{tabular}{|c|c|c|c|c|c|}
\hline $\begin{array}{l}\operatorname{Max} \\
\Delta E_{a b}\end{array}$ & $\begin{array}{c}\text { Mean } \\
\Delta E_{a b}\end{array}$ & $\begin{array}{c}\text { \# samples } \\
\Delta E_{a b}>\mathbf{4}\end{array}$ & $\begin{array}{l}\operatorname{Max} \\
\Delta E_{94}\end{array}$ & $\begin{array}{c}\text { Mean } \\
\Delta E_{94}\end{array}$ & $\begin{array}{c}\text { \# samples } \\
\Delta E_{94}>\mathbf{3}\end{array}$ \\
\hline \multicolumn{3}{|c|}{$n=1.5$} & \multicolumn{3}{|c|}{$\mathrm{n}=1.5$} \\
\hline 6.30 & 2.44 & 81 & 4.76 & 1.55 & 32 \\
\hline 5.90 & 1.90 & 38 & 3.67 & 1.21 & 11 \\
\hline 4.19 & 1.44 & 2 & 3.01 & 0.95 & 1 \\
\hline \multicolumn{3}{|c|}{$n=1.6$} & \multicolumn{3}{|c|}{$n=1.6$} \\
\hline 7.54 & 3.29 & 240 & 5.20 & 2.05 & 121 \\
\hline 7.61 & 2.79 & 139 & 4.58 & 1.73 & 67 \\
\hline 4.89 & 1.54 & 7 & 2.91 & 0.99 & 0 \\
\hline \multicolumn{3}{|c|}{$\mathrm{n}=1.7$} & \multicolumn{3}{|c|}{$n=1.8$} \\
\hline 8.49 & 3.67 & 454 & 5.77 & 2.32 & 176 \\
\hline 7.79 & 3.02 & 333 & 5.58 & 1.92 & 100 \\
\hline 6.28 & 2.67 & 278 & 4.41 & 1.72 & 67 \\
\hline
\end{tabular}


TABLE 3. Prediction accuracy for ink jet prints (Canon BJC 8500, 600 dpi)

\begin{tabular}{|c|c|c|c|c|c|c|}
\hline $\begin{array}{c}\text { Ink-jet prints (Canon BJC-8500) } \\
729 \text { test samples }\end{array}$ & $\begin{array}{l}\operatorname{Max} \\
\Delta E_{a b}\end{array}$ & $\begin{array}{l}\text { Mean } \\
\Delta E_{a b}\end{array}$ & $\begin{array}{c}\text { \# samples } \\
\Delta E_{a b}>\mathbf{4}\end{array}$ & $\begin{array}{l}\operatorname{Max} \\
\Delta E_{94}\end{array}$ & $\begin{array}{c}\text { Mean } \\
\Delta E_{94}\end{array}$ & $\underset{\Delta E_{94}>\mathbf{3}}{\# \text { samples }}$ \\
\hline 50 lpi & \multicolumn{3}{|c|}{$n=2.5$} & \multicolumn{3}{|c|}{$n=2.8$} \\
\hline Single ink dot-gain only & 10.12 & 3.60 & 262 & 6.77 & 2.60 & 284 \\
\hline $\begin{array}{l}\text { Single ink dot gain and ink spreading } \\
\text { according to print order }\end{array}$ & 8.76 & 2.97 & 196 & 5.66 & 1.97 & 160 \\
\hline $\begin{array}{l}\text { Single ink dot gain and ink spreading for } \\
\text { all superposition conditions }\end{array}$ & 3.51 & 1.30 & 0 & 2.39 & 0.84 & 0 \\
\hline 75 lpi & \multicolumn{3}{|c|}{$n=4.6$} & \multicolumn{3}{|c|}{$n=5.6$} \\
\hline Single ink dot-gain only & 11.56 & 4.44 & 387 & 8.05 & 3.22 & 398 \\
\hline $\begin{array}{l}\text { Single ink dot gain and ink spreading } \\
\text { according to print order }\end{array}$ & 10.64 & 3.61 & 260 & 6.46 & 2.37 & 236 \\
\hline $\begin{array}{l}\text { Single ink dot gain and ink spreading for } \\
\text { all superposition conditions }\end{array}$ & 3.93 & 1.30 & 0 & 2.47 & 0.83 & 0 \\
\hline 100 lpi & \multicolumn{3}{|c|}{$n=8.1$} & \multicolumn{3}{|c|}{$\mathrm{n}=12$} \\
\hline Single ink dot-gain only & 11.22 & 4.48 & 384 & 7.77 & 3.36 & 418 \\
\hline $\begin{array}{l}\text { Single ink dot gain and ink spreading } \\
\text { according to print order }\end{array}$ & 9.96 & 3.43 & 237 & 6.27 & 2.29 & 217 \\
\hline $\begin{array}{l}\text { Single ink dot gain and ink spreading for } \\
\text { all superposition conditions }\end{array}$ & 4.33 & 1.34 & 2 & 2.91 & 0.83 & 0 \\
\hline
\end{tabular}

